\title{
Exponential Decay Lifetimes of Excitons in Individual Single-Walled Carbon Nanotubes
}

\author{
Axel Hagen, ${ }^{3}$ Mathias Steiner, ${ }^{1}$ Markus B. Raschke, ${ }^{2}$ Christoph Lienau, ${ }^{2}$ Tobias Hertel, ${ }^{4}$ Huihong Qian, ${ }^{1}$ \\ Alfred J. Meixner, ${ }^{1}$ and Achim Hartschuh ${ }^{1, *}$ \\ ${ }^{1}$ Department of Physical Chemistry, Universität Siegen, 57068 Siegen, Germany \\ ${ }^{2}$ Max-Born-Institut für Nichtlineare Optik und Kurzzeitspektroskopie, 12489 Berlin, Germany \\ ${ }^{3}$ Department of Physical Chemistry, Fritz-Haber-Institut der Max-Planck-Gesellschaft, 12489 Berlin, Germany \\ ${ }^{4}$ Department of Physics and Astronomy, Vanderbilt University, Nashville, Tennessee 37235, USA
}

(Received 13 April 2005; published 31 October 2005)

\begin{abstract}
The dynamics of excitons in individual semiconducting single-walled carbon nanotubes was studied using time-resolved photoluminescence (PL) spectroscopy. The PL decay from tubes of the same $(n, m)$ type was found to be monoexponential, however, with lifetimes varying between less than 20 and 200 ps from tube to tube. Competition of nonradiative decay of excitons is facilitated by a thermally activated process, most likely a transition to a low-lying optically inactive trap state that is promoted by a lowfrequency phonon mode.
\end{abstract}

PACS numbers: 78.67.Ch, 78.47.+p, 78.55. $-\mathrm{m}$

Since the discovery of band gap photoluminescence (PL) [1], semiconducting single-walled carbon nanotubes (SWNTs) emerged as model systems for quasi-onedimensional quantum wires with great promise for applications in photonics, optoelectronics, and nanoelectronics. Among the obstacles towards a widespread use in optical devices appears to be the low photoluminescence quantum yield which, being on the order of $10^{-3}$ to $10^{-4}$ [1,2], indicates that the excited state relaxation is dominated by very efficient nonradiative decay channels for the studied SWNTs sample material. Trapping at impurity sites or at structural defects as well as branching into optically inactive excitons is discussed as competing processes to radiative recombination [2-5]. However, the detailed mechanism of nonradiative decay, its kinetics, and specifically the character and role of impurities remain unclear [2].

Time-domain studies of optical transients are powerful tools to clarify the microscopic mechanisms and the dynamics of radiative and nonradiative decay phenomena. Recently several studies of SWNT ensembles have revealed single- or multiexponential decay dynamics with time-constants ranging from 5 to 120 ps [2,3,6-8], which is orders of magnitude shorter than the radiative lifetime of $\approx 110 \mathrm{~ns}$ estimated from calculated exciton oscillator strengths [2]. Ambiguities arising from the interpretation of ensemble measurements with contributions from different tube species, nonresonant optical transients, as well as the sample heterogeneity regarding length and impurity distributions thus call for an investigation on the single tube level. Single tube PL measurements avoid the averaging of decay dynamics in ensembles of different structures $(n, m)$ and within subensembles of tubes having the same $(n, m)$ for which nonuniform emission properties have been observed for micelle encapsulated tubes $[6,9,10]$. Similarly, a distribution of excited state lifetimes and PL quantum yields may be expected even for a single tube species. Studying the properties of these distributions for SWNTs with identical $(n, m)$ therefore provides insight into the actual role of defects and allows one to distinguish between effects specific to the tube structure and external factors.

In this Letter we report the first time-resolved photoluminescence measurements on individual $(6,4)$ SWNTs in the temperature range between 48 and $182 \mathrm{~K}$. All observed PL transients were monoexponential with lifetimes ranging from less than 20 to $200 \mathrm{ps}$ for a temperature of $87 \mathrm{~K}$. Upon cooling, the lifetimes continuously increased but saturated at about $60 \mathrm{~K}$, indicative of a thermally activated nonradiative decay channel. An analysis of the temperature dependence of spectra and PL decays suggests that the observed tube-to-tube variation of PL lifetimes can be attributed to a variation of impurity or defect concentrations.

Time-correlated single-photon counting is used in combination with confocal microscopy to study the PL dynamics from individual SWNTs which were spin coated from an aqueous suspension onto glass-coverslip substrates. SWNTs used in this study were synthesized by the HiPCO technique [11] and were stabilized as individual species in aqueous suspension using sodium dodecyl sulphate (SDS) as a surfactant [1]. A typical tapping-mode atomic force microscope image [Fig. 1(a)] of such a film reveals step and terrace heights that are consistent with SDS bilayers left behind once water from the suspension has evaporated. This shows that spin-coated SWNTs used here are still in close contact with and partially imbedded in surfactant molecules from the suspension.

Optical excitation of semiconducting SWNTs at $800 \mathrm{~nm}$ is provided at $80 \mathrm{MHz}$ repetition rate by laser pluses of $\approx 200$ fs duration. PL transients were acquired using a thermoelectrically cooled IR-enhanced photomultiplier (detection range 500-910 nm) together with a timecorrelated single-photon counting board. The overall time 


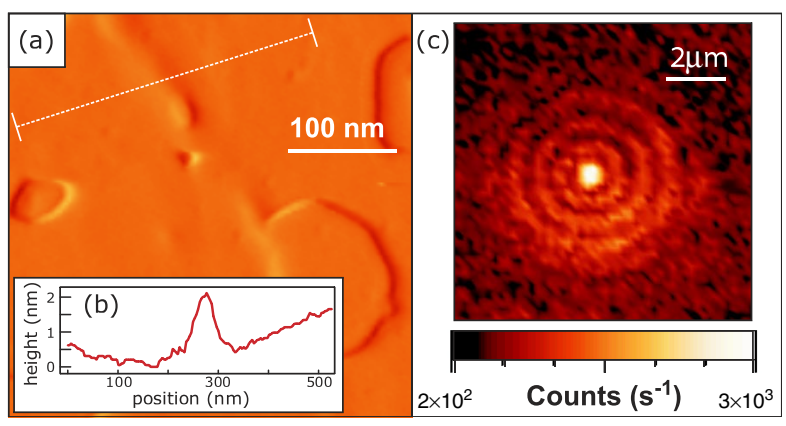

FIG. 1 (color online). (a) Atomic force microscope image of a spin-coated film containing a single SWNT on glass and (b) cross sectional profile. (c) Confocal PL image of a region around a $(6,4)$ tube. The diffraction-limited central spot at the location of the $(6,4)$ tube is about $700 \mathrm{~nm}$ in diameter.

response $\Delta \tau$ of the system was measured independently by detecting the scattered pump laser light from the sample and had a width of about 60 ps (FWHM).

The semiconducting $(6,4)$ tubes studied here were located with a low-temperature microscope by confocal imaging of the PL signal after spectral filtering by a narrow bandpass filter centered at $880 \mathrm{~nm}$, the energy of the $E_{11}$ transition of the $(6,4)$ tube [12]. Because of their small diameter, emission from $(6,4)$ tubes can readily be distinguished from neighboring tube bands at 830 and $912 \mathrm{~nm}$ corresponding to transitions in the $(5,4)$ and $(9,1)$ SWNTs, respectively [12]. A typical confocal microscope image is shown in Fig. 1(c). The width of the bright spot of $700 \mathrm{~nm}$ indicates that the size of the PL source is below the diffraction limit, which is consistent with SWNT length distributions measured for such samples [2].

A rough estimate of the single tube PL quantum yield can be obtained by comparison of the number of PL photons with the photoabsorption cross section. We estimate [13] a yield of about $10^{-4}$, indicating that the PL decay in individual tubes is indeed dominated by rapid nonradiative decay.

Figure 2 shows PL transients taken at $87 \mathrm{~K}$ [panels (a) and (b)] and $67 \mathrm{~K}$ [panel (c)] and the corresponding emission spectra [panels (d)-(f)] for three different $(6,4)$ tubes. The decay times $\tau$ of 183,60 , and 24 ps derived from a monoexponential fit are representative for the variation of PL lifetimes observed in this study with individual values spanning nearly an order of magnitude. The average of PL transients for 15 different $(6,4)$ tubes yields a lowtemperature $(T=87 \mathrm{~K}) \mathrm{PL}$ decay time of $58 \mathrm{ps}$. The distribution of $\tau$ is shown in the bar graph of Fig. 2(c). This suggests that some features of the complex dynamics observed in ensemble-averaged pump-probe and timeresolved PL measurements can be attributed to averaging over species with a wide range of lifetimes [2,3]. Remarkably, all PL transients were found to decay monoexponentially, even for tubes exhibiting spectra with multiple emission lines. Moreover, the observed monoexponential ki-
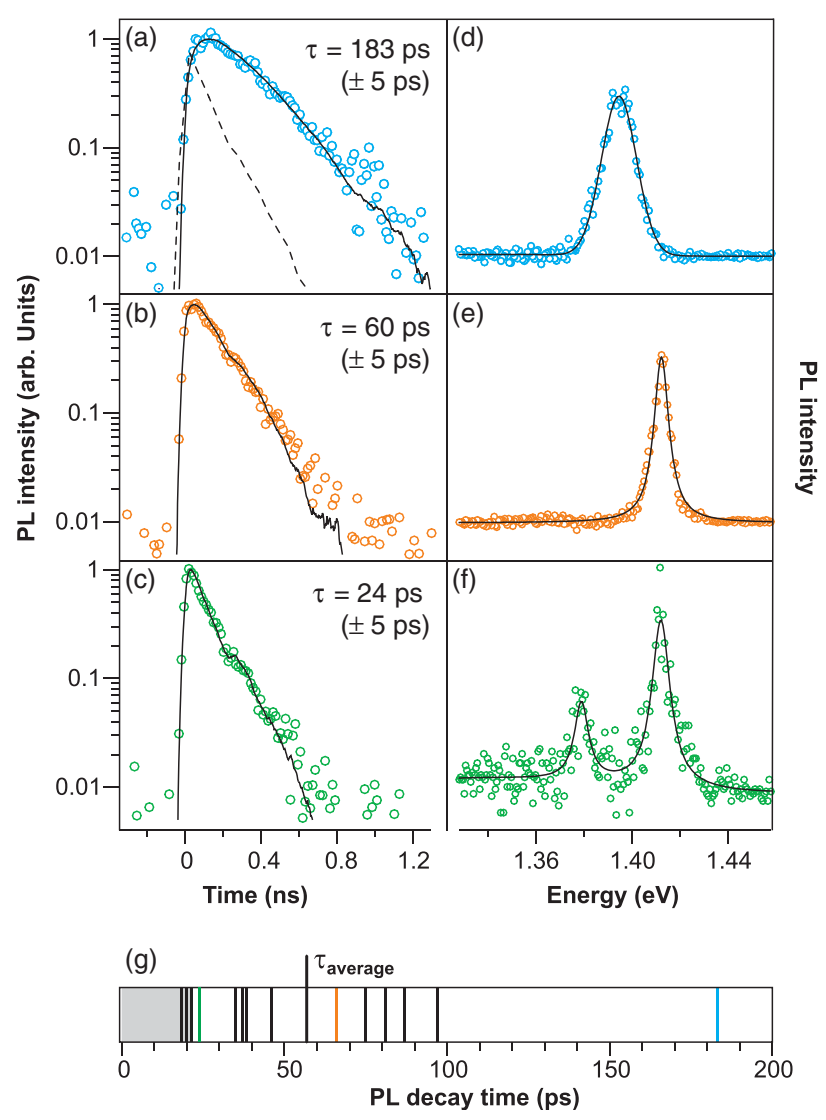

FIG. 2 (color online). Photoluminescence transients (a)-(c) and corresponding spectra (d)-(f) of three different single $(6,4)$ SWNTs observed after femtosecond pulse excitation at $800 \mathrm{~nm}$. Excited state lifetimes $\tau$ were obtained from a monoexponential fit to the experimental data [solid lines in (a)-(c)] taking into account the measured system response [dashed line in (a)]. The excitation density was $6.0 \times 10^{-4}$ photons $/\left(\right.$ pulse $\left.\mathrm{cm}^{2}\right)$ and sample temperatures were $87 \mathrm{~K}$ for (a) and (b) and $67 \mathrm{~K}$ for (c). (g) Bar graph of the distribution of measured lifetimes at $87 \mathrm{~K}$.

netics support the notion that light emission is associated with exciton decay [5,15-17] and not with free carrier recombination, which is generally expected to lead to second order (i.e., nonmonoexponential) kinetics, unless free electron and hole concentrations are significantly imbalanced. Neither the variation of the position and width nor the shape of PL spectra in Figs. 2(d)-2(f) shows any correlation with the PL transients. The low concentration of $(6,4)$ tubes in our samples of less than 1 per $500 \mu \mathrm{m}^{2}$ makes it highly unlikely that the emission lines result from more than a single $(6,4)$ tube. This indicates that spectral variations are caused by local changes in the dielectric function along individual tubes $[9,10]$.

In addition, we also studied the temperature dependence of the PL decay for individual tubes to identify the mechanism responsible for the strong tube-to-tube variations of the PL kinetics. Figure 3(a) shows the PL decay time for two different $(6,4)$ tubes as a function of temperature. The 
corresponding PL spectra for tube 1 are displayed in Fig. 3(b). For both tubes we observe a pronounced increase of $\tau$ with decreasing temperature until a constant value is reached around $60 \mathrm{~K}$. In contrast, a purely radiative exciton decay would be characterized by an increase of the PL lifetime with temperature [18]. In combination with a decrease of the PL amplitude at low temperatures the data in Fig. 3 thus confirm that $k=\tau^{-1}=k_{\mathrm{r}}+k_{\mathrm{nr}}$, i.e., the PL decay rate of individual SWNTs, is dominated by a nonradiative process, referred to by $k_{\mathrm{nr}}$. Classical Arrhenius-type kinetics for $k_{\mathrm{nr}}=\nu_{0} \exp \left(-E_{A} / k_{B} T\right)$ [dashed line in Fig. 3(a)] evidently cannot account for the saturation of lifetimes below $60 \mathrm{~K}$. Moreover, such kinetics would imply purely radiative exciton decay at $T \rightarrow 0 \mathrm{~K}$, which is clearly contradicted by the small low-temperature PL quantum yields. A quantum mechanical description including the influence of zero point vibrations on radiationless transition rates in the so-called strong coupling limit $[19,20]$ predicts $k_{\mathrm{nr}} \propto\left(k_{B} T_{\mathrm{eff}}\right)^{-1 / 2} \times$ $\exp \left(-E_{A} / k_{B} T\right)$, with $k_{B} T_{\text {eff }}=1 / 2 \hbar \omega_{m} \operatorname{coth}\left(\hbar \omega_{m} / 2 k_{B} T\right)$. Here $\omega_{m}$ is the mean frequency of the vibrational modes
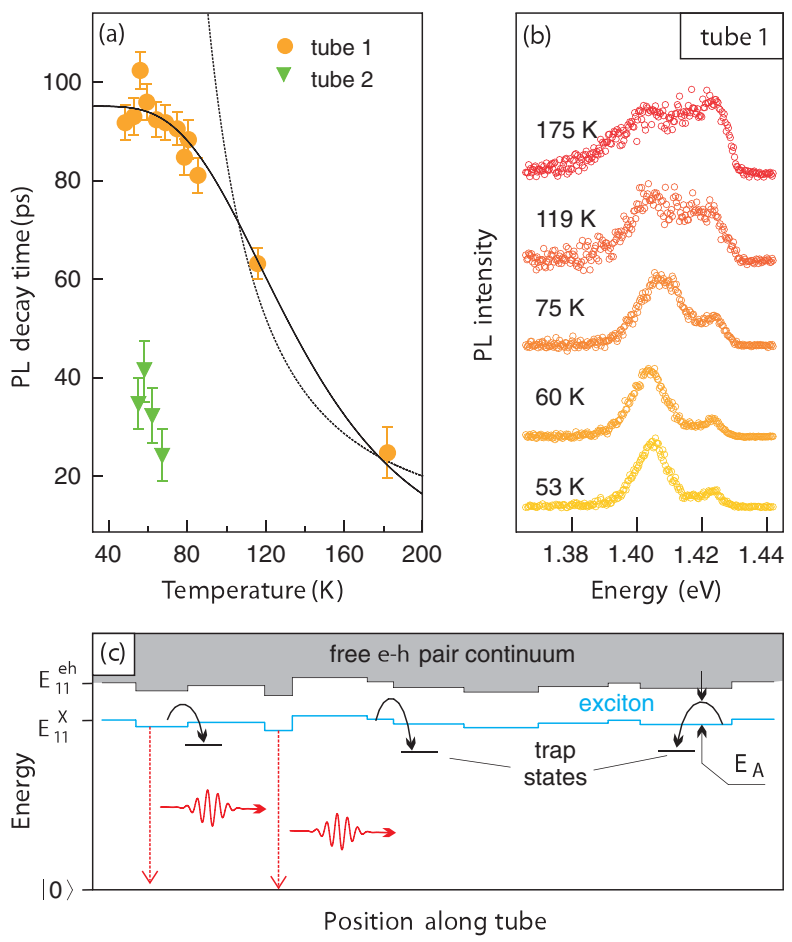

FIG. 3 (color online). (a) Temperature dependence of PL lifetimes between $48 \mathrm{~K}$ and $182 \mathrm{~K}$ for two different $(6,4)$ SWNTs. The excitation density was $4.1 \times 10^{14}$ photons $/\left(\right.$ pulse $\left.\mathrm{cm}^{2}\right)$. The solid line represents a fit using the Bixon-Jortner model $[19,20]$, while the dashed line corresponds to a simple Arrhenius fit. (b) Evolution of the emission spectrum with temperature for tube 1. (c) Schematic illustration of the potential energy landscape used to discuss the dependence of PL intensities and peak shapes as well as the kinetics of thermally activated nonradiative decay. $E_{11}^{\mathrm{eh}}$ and $E_{11}^{X}$ denote the first subband free carrier and exciton thresholds, respectively. that promote nonradiative decay [20]. This can account for both the plateau of the low-temperature decay rate and the activated behavior at higher temperatures. A fit to the experimental data yields an activation energy $E_{A}$ of $170 \pm$ $20 \mathrm{meV}$ and a mode frequency $\omega_{m}$ of $300 \pm 20 \mathrm{~cm}^{-1}$ [solid black line in Fig. 3(a)]. This coincides with the radial breathing mode of the $(6,4)$ tube, a strong low-frequency Raman mode at $337 \mathrm{~cm}^{-1}$ [21]. We thus speculate that one or several modes in this frequency range may dominate vibrationally assisted nonradiative exciton decay. PL studies of nonradiative decay in tube ensembles with different diameter are consistent with this interpretation [22].

The nonradiative decay processes studied here may either populate longer-lived trap states [2] related to defects in the tube wall [see Fig. 3(c)] or they may populate optically inactive states which are supposed to be intrinsic to the tubes' electronic structure [4]. In the latter case, variations in PL lifetime from tube to tube should be related to fluctuations of the activation energy and thus to changes in the energetic positions of the relevant electronic states. Our experiments, however, indicate that PL decay rates and the position of spectral features are determined by independent factors, making this scenario unlikely. Different concentrations of localized trap states, on the other hand, can easily account for the variations in the nonradiative exciton decay rates. In such a model, efficient excitonic transport along the tube axis is required to explain the observed monoexponential decays. Indeed, the temperature dependent spectra shown in Fig. 3(b) indicate electronic coupling and population transfer between two slightly offset emission bands. This is consistent with exciton diffusion lengths $L_{D}=\sqrt{D} \bar{\tau}$ of about $1 \mu \mathrm{m}$, i.e., more than the typical tube length [2], estimated from $D \approx 120 \mathrm{~cm}^{2} / \mathrm{s}$ [23] a lower limit to exciton mobilities and $\tau=90$ ps. The resulting scenario is illustrated schematically in Fig. 3(c). Up to now, the microscopic nature of the defect related trap states is unclear. In principal, also intrinsic, optically inactive excitonic states $[4,24]$ could serve as trap states if transitions between initially excited active states and inactive states were mediated by defects [25].

As for the variation of PL lifetimes, the powerdependence of the PL decay in different $(6,4)$ tubes is also strongly tube dependent as seen in Fig. 4. Tubes 1 and 2 exhibit a saturation of the PL amplitude as the pulse fluence $\Phi_{0}$ is increased up to $5 \times 10^{15} \mathrm{~cm}^{-2}$. This amplitude saturation is consistent with recent pump-probe studies finding pronounced photoinduced absorption bleaching at fluences in the range of $10^{14}$ to $10^{15} \mathrm{~cm}^{-2}[8,26]$. While the amplitude saturation is similar for both tubes, the power dependence of the decay time is markedly different. For tube 1 , with fairly short lifetime, it is characterized by an approximately linear decrease of $\tau$ from 50 to below 20 ps. For tube 2, with a similarly long $\tau$ as the tube studied in Fig. 3(a), $\tau$ remains high at 183 ps and is power indepen- 
(a)
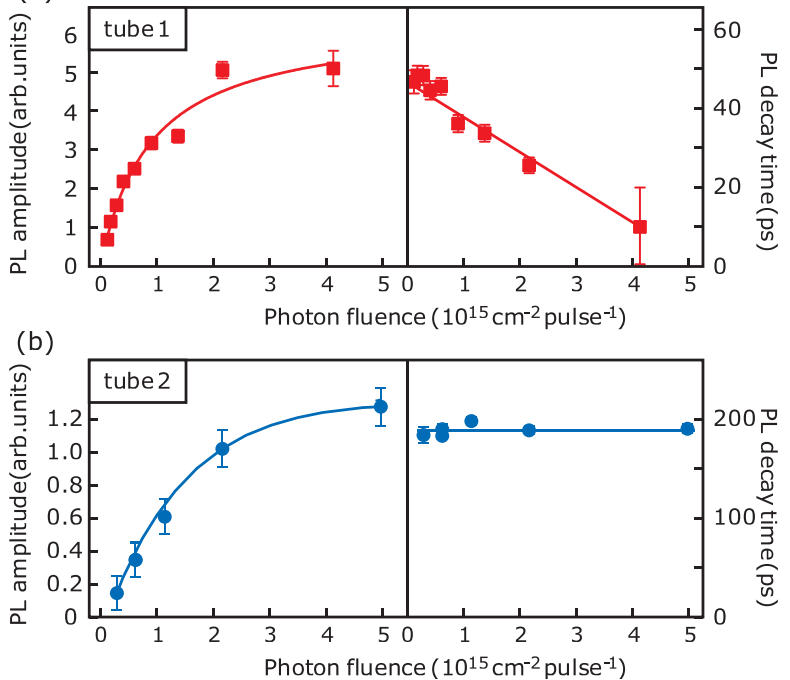

FIG. 4 (color online). Power dependence of PL amplitudes (left) and lifetimes (right) for two different $(6,4)$ SWNTs [(a), (b)] at $87 \mathrm{~K}$. Solid lines serve as a guide to the eye.

dent even at $\Phi_{0}=5 \times 10^{15} \mathrm{~cm}^{-2}$. This demonstrates that photon-induced heating of the nanotube, expected to yield a decrease in $\tau$ with increasing $\Phi_{0}$ is not the cause of the low-temperature PL decay time saturation observed in Fig. 3(a).

A power-dependent decrease of the average PL lifetime has previously been observed and ascribed to either exciton-exciton annihilation [3] or Auger recombination, possibly involving free carriers [27]. In our experiments, such processes would be characterized, for tubes with $\tau \gg$ $\Delta \tau$, by the appearance of a short-lived, nonexponential decay component at high fluences, which is not observed for tube 2. For tube 1, on the other hand, our time resolution $\Delta \tau$ is not sufficient to resolve such a nonexponential decay. Therefore, we cannot distinguish wether the reduction in $\tau$ with $\Phi_{0}$ arises from an exciton-annihilation or photoninduced heating of the nanotube. Both scenarios would explain not only the correlation between defect concentration and power dependence of the PL lifetimes but also the striking connection between the extraordinary long PL lifetime of 183 ps and the absence of any power dependence of the PL decay for tube 2.

The observed pronounced tube-to-tube variations of PL decay times and their power dependence demonstrate that the exciton dynamics in individual semiconducting single wall nanotubes is strongly influenced by defect related trap states. These results allow us to conclude that PL yields may be enhanced by at least a factor of 3 beyond the ensemble average, provided one can achieve appropriate control over extrinsic factors contributing to nonradiative decay. Further experimental and theoretical work will fo- cus on identifying the microscopic nature of these trap states and, in particular, on clarifying the role of optically inactive low-lying exciton states on the exciton dynamics which may pose an additional limitation to the PL yield.

We thank G. Schulte for experimental assistance. One of us (A. H.) thanks G. Ertl for financial support. Financial support by the DFG (SFB296) is gratefully acknowledged.

*Electronic address: achim.hartschuh@uni-tuebingen.de

[1] M. J. O'Connell et al., Science 297, 593 (2002).

[2] F. Wang, G. Dukovic, L. E. Brus, and T. F. Heinz, Phys. Rev. Lett. 92, 177401 (2004).

[3] Y.Z. Ma et al., J. Chem. Phys. 120, 3368 (2004).

[4] H. Zhao and S. Mazumdar, Phys. Rev. Lett. 93, 157402 (2004).

[5] V. Perebeinos, J. Tersoff, and P. Avouris, Phys. Rev. Lett. 92, 257402 (2004).

[6] A. Hagen, G. Moos, V. Talalaev, and T. Hertel, Appl. Phys. A 78, 1137 (2004).

[7] S. Reich et al., Phys. Rev. B 71, 033402 (2005).

[8] L. Huang, H. N. Pedrosa, and T.D. Krauss, Phys. Rev. Lett. 93, 017403 (2004).

[9] A. Hartschuh, H.N. Pedrosa, L. Novotny, and T.D. Krauss, Science 301, 1354 (2003).

[10] H. Htoon et al., Phys. Rev. Lett. 93, 027401 (2004).

[11] P. Nikolaev et al., Chem. Phys. Lett. 313, 91 (1999).

[12] S. M. Bachilo et al., Science 298, 2361 (2002).

[13] A rough estimate of single tube PL quantum yields $\eta$ can be obtained from $\eta=\sigma_{\mathrm{PL}} / \sigma_{a}=I_{\mathrm{PL}} / \sigma_{a} \Phi_{0}$. Here, $I_{\mathrm{PL}}$ is the measured number of PL photons and $\sigma_{a}$ the photoabsorption cross section. The PL cross section $\sigma_{\mathrm{PL}}$ is defined as $\sigma_{\mathrm{PL}}=I_{\mathrm{PL}} / \Phi_{0}$. For a typical incident fluence of $\Phi_{0}=6 \times 10^{14} \mathrm{~cm}^{-2}$ per pulse, a photoabsorption cross section of $\approx 1 \times 10^{-18} \mathrm{~cm}^{-2} /$ atom [14], a tube length of $400 \mathrm{~nm}$ with approximately 30000 atoms, and the PL count rate of $10^{2} \mathrm{~Hz}$ while considering the detection sensitivity of our setup of $10^{-3}$ this yields $\eta \simeq 10^{-4}$.

[14] Y. Murakami et al., Phys. Rev. Lett. 94, 087402 (2005).

[15] T. Ando, J. Phys. Soc. Jpn. 66, 1066 (1997).

[16] C. L. Kane and E. J. Mele, Phys. Rev. Lett. 93, 197402 (2004).

[17] E. Chang, G. Bussi, A. Ruini, and E. Molinari, Phys. Rev. Lett. 92, 196401 (2004).

[18] J. Feldmann et al., Phys. Rev. Lett. 59, 2337 (1987).

[19] M. Bixon and J. Jortner, J. Chem. Phys. 50, 4061 (1969).

[20] R. Englman and J. Jortner, Mol. Phys. 18, 145 (1970).

[21] M.S. Strano et al., Nano Lett. 3, 1091 (2003).

[22] T. Hertel et al., Nano Lett. 5, 511 (2005).

[23] O.J. Korovyanko et al., Phys. Rev. Lett. 92, 017403 (2004).

[24] J. Maultzsch et al. (to be published).

[25] V. Perebeinos, J. Tersoff, and P. Avouris (to be published).

[26] J.-S. Lauret et al., Phys. Rev. Lett. 90, 057404 (2003).

[27] F. Wang et al., Phys. Rev. B 70, 241403(R) (2004). 